\title{
Application of Artificial Intelligence-based Technology in Cancer Management: A Commentary on the Deployment of Artificial Neural Networks
}

\author{
GAJANAN V. SHERBET ${ }^{1}$, WAI LOK WOO ${ }^{2}$ and SATNAM DLAY ${ }^{2}$ \\ ${ }^{1}$ The Institute for Molecular Medicine, Huntington Beach, CA, U.S.A.; \\ ${ }^{2}$ School of Engineering, University of Newcastle upon Tyne, Newcastle upon Tyne, U.K.
}

\begin{abstract}
Artificial intelligence was recognised many years ago as a potential and powerful tool to predict disease outcome in many clinical situations. The conventional approaches using statistical methods have provided much information, but are subject to limitations imposed by the complexity of medical data. The structures of the important variants of the machine learning system artificial neural networks (ANN) are discussed and emphasis is given to the powerful analytical support that could be provided by ANN for the prediction of cancer progression and prognosis. The predictive ability of the cellular markers, DNA ploidy and cell-cycle profiles, and molecular markers, such as tumour promoter and suppressor gene, and growth factor and steroid hormone receptors in breast cancer management were also analysed. ANN systems have been successfully deployed to evaluate microRNA profiles of tumours which saliently sway cancer progression and prognosis of the disease, thus counteracting the negative implications of their numerical abundance. Finally, in this setting, the prospective technical improvements in artificial neural networks, as hybrid systems in combination with fuzzy logic and artificial immune networks were also addressed.
\end{abstract}

This article is freely accessible online.

Correspondence to: Gajanan V. Sherbet, The Institute for Molecular Medicine, Huntington Beach CA, U.S.A. E-mail: gsherbet@ immed.org; gajanan.sherbet@ncl.ac.uk

Key Words: Artificial neural networks, binomial logistic regression, breast cancer, DNA ploidy, Fuzzy k-nearest neighbour algorithm, Fuzzy neural networks, growth factor receptors, molecular markers, multilayer perceptron architecture, oestrogen and progesterone receptors statistical analyses, tumour progression and prognosis, tumour promoter and suppressor genes, review.
Breast cancer is a most frequent cause of cancer death in women. Understandably much attention has been focused on treatment and patient management. The current criteria that determine patient management relate to the state of progression of the disease. The histological grade, tumour stage and vascular and lymphatic invasion and hormone receptor status and combinations of these are employed to identify risk groups and to predict prognosis $(1,2)$. However, there is an acute need to identify fresh innovative approaches and adapt the available technology to determine the prognosis of individual patients. Numerous molecular markers are known that individually or in combination relate to the state of the disease. Several molecular parameters are known to influence tumour growth, dissemination and progression $(3,4)$, but the utilisation of these to extract clinically valuable prognostic information and to determine the course of the disease and prognosis for individual patients is fraught with difficulties.

The major hurdle is the numerical abundance of markers. Assessing the clinical value of individual markers or assigning them predictive weight is an onerous task. This would be affected by the influence they would exert as single markers. Their predictive credence could be at pronounced variance while functioning in combination with others on account of the crosstalk of the signalling systems activated by the markers. This crosstalk would reflect in the final outcome. It is in this area of uncertainty and ambiguity that the neural networks come to the fore.

\section{Artificial Neural Networks in Cancer Studies}

The conventional statistical approaches of linear regression and logistic regression have provided valuable information relating to disease states, but their utility is subject to limitations imposed by the complexity of medical data. In recent years machine learning ANN and fuzzy approaches such as FK-NN (Fuzzy K-nearest neighbour) have provided 
much analytical support to predict the aggressive behaviour of tumour and prognosis of the disease. Artificial intelligence technology in the form of ANN has been extensively used in assessing the degree of aggressive activity of cancers and has impressively aided to devise novel approaches to predict the course of the disease and prognosis and in this way provide potential guidelines to determine modes of treatment.

The use of artificial neural networks and variants dates back many years. The structure and function of ANN largely mimics the brain. It is composed of perceptrons that functionally simulate the neurons. Both neural networks and fuzzy systems mimic the brain, but a vibrant distinction may be made between the two systems. The neural networks manipulate well-defined confined data. In contrast, fuzzy systems can handle and make decisions on data sets with undefined indeterminate boundaries and the decisions rely on degrees of differences in different classes of objects since they are not defined by sharp boundaries unlike the Boolean true and false binary decisive logic. Fuzzy systems have been regarded as sound and beneficial alternatives to ANN and statistical methods. The FK-NN is a potent pattern classier $(5,6)$. The nearest neighbour classification involves identification of similar $\mathrm{k}$ patterns in the training data set and formulates a pattern which is tested for its occurrence across the unseen data. The training needs to be carried out for every analytic session and cannot be stored for subsequent use for classifying a new set of data. The algorithm has seen many improvements, some postulated and others implemented, in the structure of the patterns in order to achieve superior classification. The FK-NN has been successfully deployed for breast cancer studies $(7,8)$.

\section{Basic Architecture of ANNs}

The basic concept is establishing a decision-making unit with inter-connection of the perceptron units and this enables nonlinear analysis (Figure 1). There are two types of ANN. Among the most commonly utilised ANN formats are the multilayer perceptron (MLP). This represents a feed-forward network wherein a layer of input perceptrons connects to a number of hidden layers of perceptrons followed by an output layer. This model has been used in several clinical studies to predict the risk of mortality of stroke patients and the risk of haemorrhage in ischemic stroke $(9,10)$ and for diagnosing cardiovascular diseases $(11,12)$. The MLP has been shown to be an effective and reliable vehicle for exploring the predictive potential of some established biomarkers for cancer (13).

\section{Application of ANNs as a Non-invasive Tool to Determine Tumour Dissemination}

Our main aim has been directed at identifying robust noninvasive surrogate methods that can predict the potential metastatic spread of cancer. Among notable putative molecular markers employed for this purpose are S100A4 tumour promoter gene and the anti-metastasis gene $\mathrm{nm} 23$. In several tumour types the expression levels of S100A4 has shown positive correlation with cell proliferation and the expression of growth factor receptors and might represent positive responses to stimuli from growth factors (14-17). Many studies have demonstrated that high expression of the nm23 gene or its product the NDP (nucleoside diphosphate) kinase consistently correlated with low metastatic potential. In breast cancer nm23 expression was inversely related to nodal spread and survival (18-20).

In a study of breast cancers, data relating to the expression of cell and molecular markers viz. tumour grade/size, expression of ER (oestrogen receptor/PR (progesterone receptor), and the tumour promoter S100A4 and metastasis suppressor nm 23 genes were provided to the input neurones. The layers of neurons determine the non-linear relationships in the input patterns. The output neuron provides the outcome in terms of the presence of the tumour in the regional lymph nodes. This study showed that the relative expression of S100A4 and nm23 genes was the single most effective predictor of nodal status. Tumour grade and size seemed to have no influence on prediction, but the inclusion of ER/PR with grade and size markers notably improved prediction $(21,22)$ suggesting that the hormone receptors were exerting complementary pressures (Figure 2).

A most pertinent problem in the context of designing therapeutic strategy is establishing the possibility of the development of resistance or sensitivity to endocrine therapy and the identification of subgroups of patients likely to benefit from the therapy. Caronongan III (23) recently addressed the issues concerning the evaluation of the relative potential of conventional hormonal markers, which they deemed were essential to evolve new molecular approaches to breast cancer management. From this study a clear distinction emerged between the relative importance of PR and ER in breast cancer. Notable was that PR displayed greater correlation than ER with disease progression and prognosis. The obvious implication here is that there is need to expand the current practice of focusing solely on ER expression to include PR assessment whilst determining adjuvant treatment. To consolidate the comments, one could say that there is little doubt that ANN is a significant methodology for the analyses of molecular markers to predict prognosis. This has been shown to be the case also in oral cancers. Chang et al. (24) showed that machine-based analyses of pathological markers and ambient molecular features were most productive.

These studies underscore the power of ANN techniques to predict the aggressive behaviour of breast cancer which has provided overwhelming impetus to pursue analyses along more sophisticated routes. ER and PR are closely linked with breast cancer progression. Their expression status is significant in determining response to endocrine therapy. ER signalling engages in crosstalk with EGFR (epidermal growth factor receptor) and HER2 (human epidermal growth factor receptor 

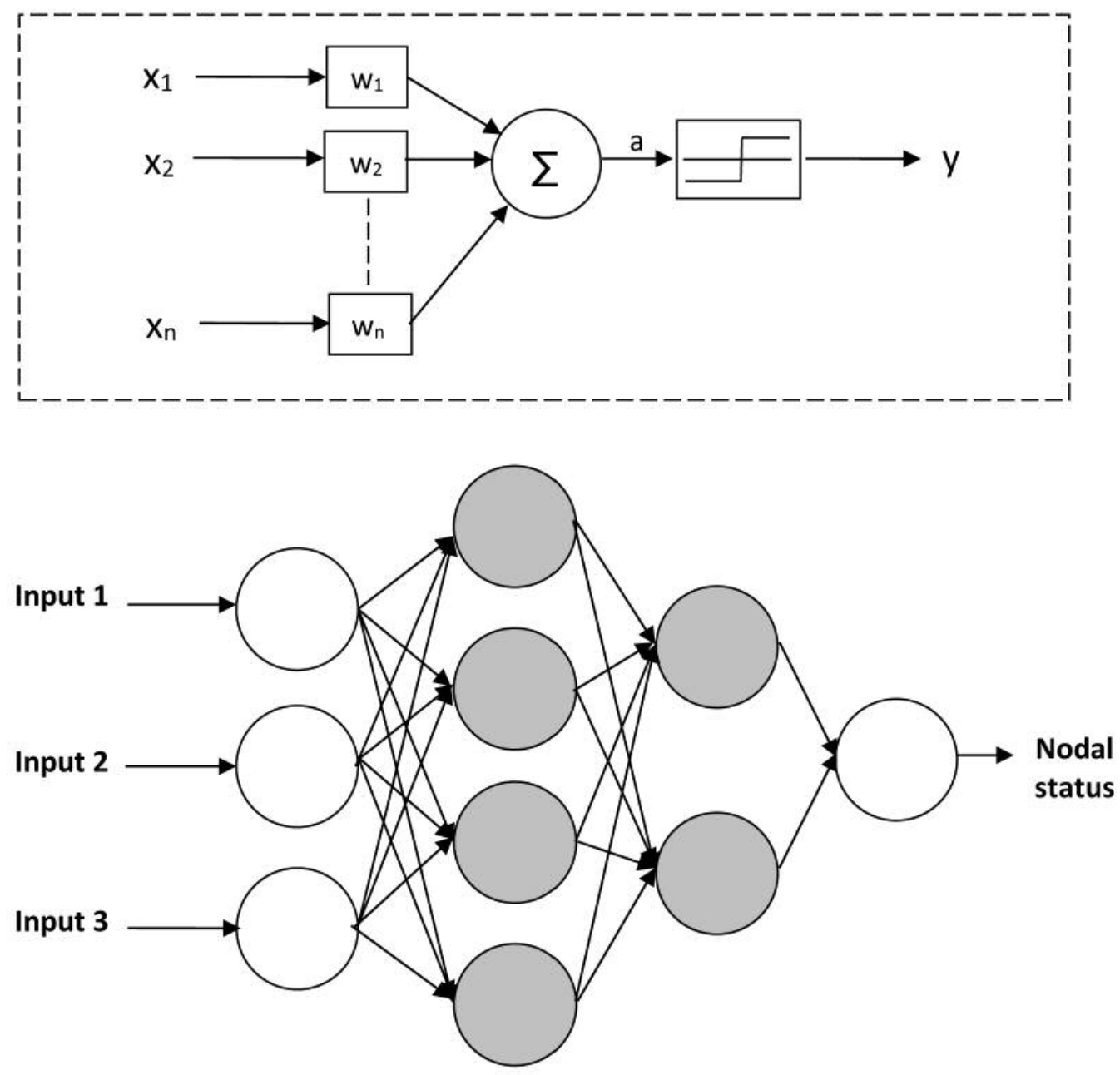

Figure 1. A multilayer perceptron (MLP) feed forward network is shown. Each circle represents one perceptron, as shown in more detail in the dashed box at the top. This configuration represents just one of any number of possible layouts. The grey cells represent the hidden layers of the network. In the studies discussed here the inputs are follows. Input 1: cell proliferation markers, input 2: Histology features and 3 are molecular markers. The output is frequency of nodal involvement. Figure modified from (22).

2). As alluded to already, there is obvious interaction of steroid receptors signalling with signalling pathways activated by the tumour promoter S100A4 and the suppressor nm 23 genes. The abnormal growth kinetics of cancer cells is reflected in the DNA indices, aberrant gene amplification and expression and the size of the DNA replicating or synthesis phase fraction (SPF). Hence, we have posed the question whether these factors also influence the function of ER/PR by serving as complementary components to predict prognosis of breast cancer.

\section{Binomial Regression Analysis of Cancer Markers}

The abnormal growth kinetics of cancer cells is reflected in the DNA indices which are related to DNA ploidy, the size of the fraction of cells in the DNA replicative phase of the cell cycle, and aberrant gene amplification and expression. Binomial logistic regression algorithm, MLP and FK-NN classifiers have been employed in many studies to check if the DNA markers aided the prediction of nodal status and survival of breast cancer patients. The binomial regression algorithm has been frequently employed to predict aggressive behaviour of prostate cancers. Tsao et al. (25) have claimed to be able to predict the clinical stage of prostate cancer indicated by Gleason grade and prostate specific antigen levels. They used both logistic regression algorithm and neural networks in this study and have described ANNs as more powerful than the statistical tool to predict potential capsular invasion by the cancer. A study of hepatocellular carcinoma employing ANN and regression analyses also revealed ANN to be a more powerful tool for predicting patient survival (26). 


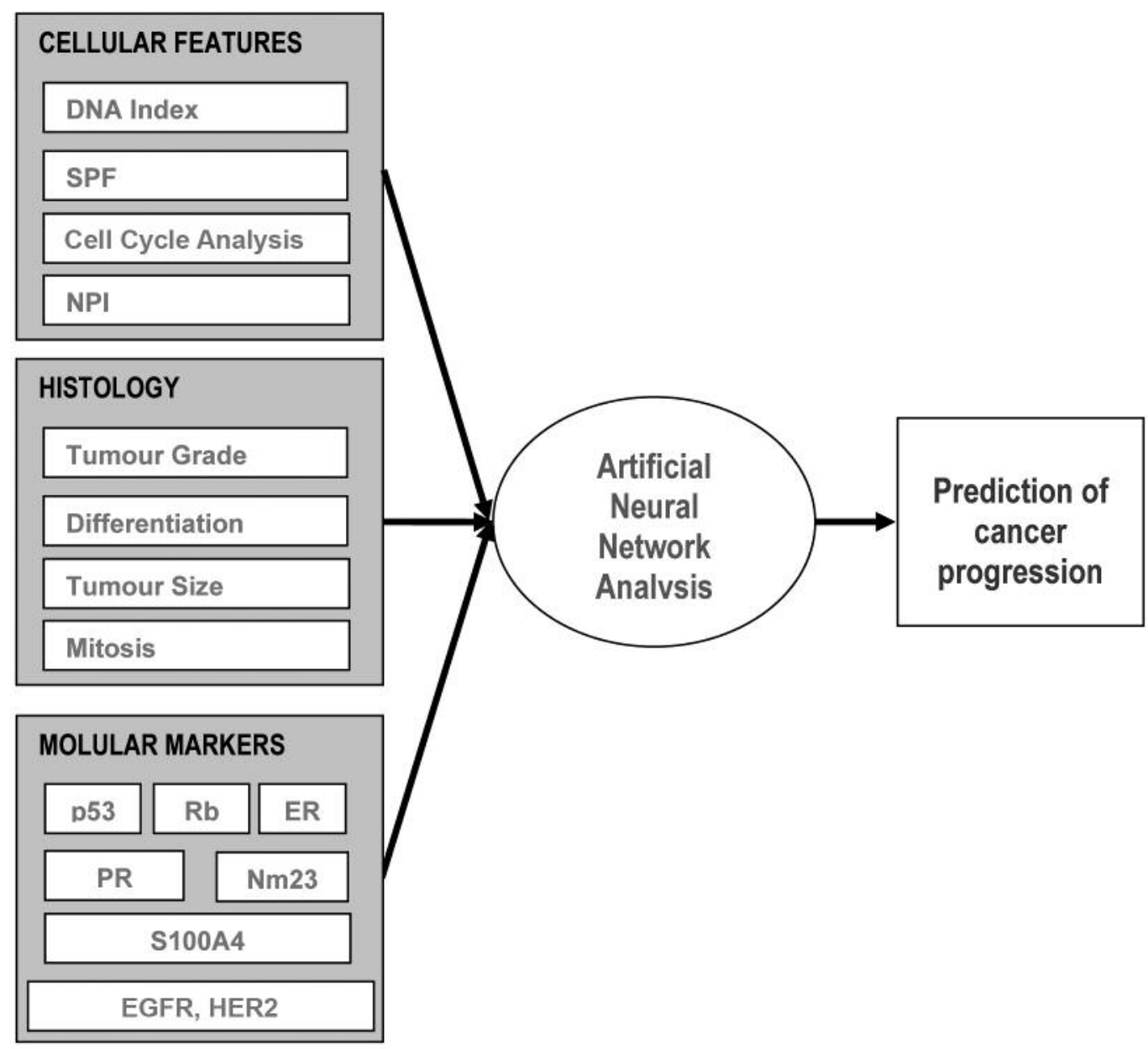

Figure 2. The flow of data through a system based on artificial neural network is shown here. The grey boxes show a few of the possible inputs into the system. This figure is modified from Grey et al. (22). See discussion below. EGFR: Epidermal growth factor receptor; ER: oestrogen receptor; HER1: Human EGFR1; NPI: nuclear pleomorphism index; PR: progesterone receptor; SPF: S-phase fraction.

DNA ploidy has often constituted a part of the general picture of tumour progression and prognosis. Using the logistic regression algorithm, Andronas et al. (27) demonstrated the possibility of predicting nodal involvement and 5-year disease-free survival by combining ER/PR status with DNA ploidy, SPF and $\mathrm{G}_{0} \mathrm{G}_{1} / \mathrm{G}_{2} \mathrm{M}$ ratios. They recorded a high degree of accuracy for predicting both nodal involvement and 5-year disease-free survival. Higher DNA index and lower $\mathrm{G}_{0} \mathrm{G}_{1} / \mathrm{G}_{2} \mathrm{M}$ ratios of cell-cycle profile, both features reflecting aneuploidy, were predictive of prognosis $(28,29)$. Indeed, more recent studies have confirmed the value of including DNA ploidy in the assessment of prognosis. DNA ploidy and SPF both act as complementary factors and accentuate the predictive power of ER/PR for breast cancer progression and provide credibility that they could deliver a more reliable prognostic model to assist in patient management (23, 30). Nevertheless, supportive evidence was obtained by analysing the data set of Andronas et al. (27) aided by more sophisticated analyses, viz. by using the FK-NN algorithm. Data containing histology type, tumour grade, DNA ploidy, S-phase fraction, $\mathrm{G}_{0} \mathrm{G}_{1} / \mathrm{G}_{2} \mathrm{M}$ ratio, and nuclear pleomorphism indices from one hundred breast cancer specimens and benign conditions were analysed for the prediction of nodal involvement and 5-year survival. The fuzzy method FK-NN technique yielded the highest predictive accuracy of $88 \%$ for both nodal involvement and survival analyses in the subset which considered all the cellular markers. The predictive performance was far superior in comparison with the statistical logistic regression method and multilayer feed forward backpropagation neural networks (8). 


\section{ANN Analyses of microRNA Expression to Predict Cancer Prognosis}

A species of genomic markers that have come to the fore are the miRNAs (microRNAs). A large number of non-coding microRNAs have been associated with cancer. The expression of some of them has paralleled cancer progression. Some miRNAs seem to function as tumour promoters, whilst others appear to be suppressors of cancer progression. However, assigning specificity of action that could lead to the promotion or suppression of cancer has been an onerous task on account of the numerical magnitude of microRNAs. Furthermore, long non-coding RNAs can encode miRNAs and function as precursors of miRNAs. This, in turn, can result in complementary or competitive biological behaviour between the long non-coding RNAs and miRNAs.

It is of considerable interest that notwithstanding these caveats much effort is going into the deployment of ANN systems to evaluate miRNA profiles of tumours to identify which miRNAs saliently influence cancer progression and prognosis of the disease. ANN analyses have revealed a profile of miRNAs found in the serum of ovarian cancer patients. With ANN systems trained using an algorithm for pattern recognition of the miRNA prolife it was possible to diagnose ovarian cancer more efficiently than achieved by conventional markers such as CA125. Furthermore, the finding that the same miRNAs were also expressed in the tumours has provided much support for the potential use of the technology in the diagnosis of the disease (31). CA125 is not an infallible marker of ovarian cancer. Normal subjects can display higher CA125 levels. In fact, the marker is not specific for ovarian cancer and enhanced levels of the marker are encountered in patients with other gynaecological cancers and also in benign endometriosis. Raised CA125 levels also occur in breast, colon and lung cancers. However, higher CA125 levels may correlate with progression and metastatic spread (32-34). Analysis using ANN of miRNA expression profiles of nasopharyngeal carcinoma has allowed identification of a small number of miRNA that appeared to be predictive of death risk. The miRNA expression values transformed into miRNA scores correlated with survival status of the patients (35). At any rate, ANN analyses might complement and even overcome some of the uncertainties linked with the significance of CA125 in cancer management.

The miRNAs bind to the 3'-UTR (untranslated region) and regulate the expression of target genes. The prediction of this interaction and detection of the target genes appears to be a distinct possibility and within the ambit of appropriately trained ANNs $(36,37)$. However, the practical application of this methodology to the identification of genes regulating the development and progression of cancer and whose expression is modulated by miRNA remains to be established.

\section{Fuzzy Neural Systems}

The fuzzy neural system developed some years ago incorporates a neural model with fuzzy synapses and learning algorithms which adjust weights of the synaptic connections. Hierarchical fuzzy neural networks and fuzzy Gaussian potential neural networks have successfully classified benign and malignant breast cancer lesions, not a remarkable achievement in itself. Hierarchically connected fuzzy neural systems do use fewer rules to analyse non-linear data sets with high accuracy. The structure and training of the networks are said to be difficult to establish. The analytical accuracy of hierarchical fuzzy neural networks would depend upon the incorporation of optimum number of perceptrons and the fuzzy rules governing interaction between them. Nonetheless, the combination of neural networks and fuzzy logic has turned out to be highly effective for dealing with non-linear systems (38-41).

The multi-layered perceptron system with its fuzzy synapses is trained to provide the desired decisions (42). The trained system can then be used to predict the outcome from unseen input data. Neural networks and fuzzy logic have been combined to develop some hybrid models. The fuzzyCSFNN (conic section function neural network) with MLP and radial basis function (RBF), Fuzzy-MLP and Fuzzy-RBF structures have been tested for their performance for thyroid and breast cancer prognosis (43). The RBF units respond to the input data by Gaussian regression. The application of Gaussian function network to analyse non-linear input data was mooted many years ago (44). The training algorithm integrates that analytical ability to the requisite hidden Gaussian units and thus it holds an advantageous position compared with the back-propagation training of MLP.

The Gaussian process is akin to a hidden two-layer perceptron neural network. In MLP, the addition of hidden layers progressively enhances the learning ability of the system. Likewise, addition of more layers to neural fuzzy hybrid models is employed to analyse non-linear input data. It has been argued that the learning algorithm and the back propagation of MLP are not consistent biologically with the requirement of bidirectional functioning of the synapses. The contrasting view is that the Gaussian regression is not based on biological models and does not require prolonged training. The addition of extra perceptron layers would provide the Gaussian regression approach with enhanced predictive power (45).

\section{Combination of ANN and Artificial Immune Networks}

Recently, a two-stage process where ANN was combined with an artificial immune network has been suggested. This hybrid system appears to have led to improvements in the prediction of prognosis (46). The artificial immune system imitates the 
biological features of ligand and receptor interaction, viz. ligand binding specificity and binding affinity to the relevant receptors. This algorithm constructed on biological parameters can be employed to train and optimise the functional output of the hidden neurons of the ANN. Whilst neural networks depend upon learning of optimal amounts of observed data which train the hidden black box neurons, a fuzzy neural network needs no previously known data and can function with the neural network structure allied to the optimising function of the artificial immune network (47-50). This immune network algorithm for function optimisation possibly results in the improvements in the prognostic predictions achieved by Kuo et al. (46).

\section{Conclusion}

Artificial intelligence was recognised as a powerful tool to analyse inter-relationships between numerous variables within medical data from several fields of medicine, aimed overall at extracting information that could lead to diagnosis, treatment and predicting prognosis of several disease states. The accent here is on the role of the several variants of artificial neural networks in cancer management and prognosis. The application of ANNs as a non-invasive tool to determine tumour dissemination and development of metastatic disease using the expression of tumour promoter and suppressor genes as molecular markers was discussed. Also, it was shown how ANN reveals the relative significance of growth factors and steroid hormone receptors in the prediction of breast cancer prognosis. The capability of ANNs to uncover the influence of DNA ploidy and cell-cycle profiles on the predictive competence of the hormone receptors was also demonstrated. The deployment of ANN systems to evaluate miRNA profiles of tumours to identify the miRNAs which saliently influence cancer progression and prognosis of the disease has taken significant positive strides. This has counterbalanced beyond measure the disadvantages arising from the numerical abundance of miRNAs that has eclipsed their potential in cancer development and progression. The prospective technical improvements in artificial neural networks was also addressed. The structures of the important variants of ANN were emphasised with accrued benefits as non-invasive tools to assess disease prognosis. ANN hybrid systems such as neural networks and fuzzy logic, and ANN systems combining the neural architecture with artificial immune networks have led to considerable improvements in predictive potential relevant in the context of cancer management.

\section{Conflicts of Interest}

GVS confirms on behalf of all the Authors that there are no conflicts of interest in regard to this work.

\section{References}

1 Sherbet GV: The biology of tumour malignancy, Academic Press, London, 1982.

2 Sherbet GV: Preface. Molecular and cellular pathology of cancer progression and prognosis. Ed. Sherbet GV, Research Signpost, Trivandrum, 2006.

3 Sherbet GV: Growth factors and their receptors in cell differentiation, cancer and cancer therapy, Elsevier, 2011.

4 Sherbet GV: Molecular approach to cancer management. Elsevier/Academic Press, 2017.

5 Szczepaniak PS, Lisboa PJG and Kacprzyk J: Fuzzy Systems in Medicine, Physica-Verlag, A Springer-Verlag Company, 2000.

6 Kuncheva LI: Fuzzy classifier design, Heidelberg: SpringerVerlag, 2000.

7 Li Q, Li W, Zhang and Xu Z: An improved k-nearest neighbour method to diagnose breast cancer. Analyst 143: 2807-2811, 2018.

8 Seker H, Odetayo MO, Petrovic D, Naguib RNG, Bartoli C, Alasio L, Lakshmi MS and Sherbet GV: Assessment of nodal involvement and survival analysis in breast cancer patients using image cytometric data: statistical, neural network and fuzzy approaches. Anticancer Res 22: 433-438, 2002.

9 Süt N and Çelik Y: Prediction of mortality in stroke patients using multilayer perceptron neural networks. Turkish J Med Sci 42: 886-893, 2012.

10 Bentley P, Ganesalingam J, Jones ALC, Mahady K, Epton S, Rinne P, Sharma P, Halse O, Mehta A and Rueckert D: Prediction of stroke thrombolysis outcome using CT brain machine learning. Neuroimage Clin 4: 635-640, 2014.

11 Yan H, Jiang Y, Zheng J, Peng C and Li Q: A multilayer perceptron-based medical decision support system for heart disease diagnosis. Expert Sys Apps 30: 272-281, 2006.

12 Panday SP and Godara N: Decision support system for cardiovascular heart disease diagnosis using improved multilayer perceptron. Int J of Computer Appl 45: 12-20, 2012.

13 Mojarad SA, Dlay SS, Woo WL and Sherbet GV: Cross validation evaluation for breast cancer prediction using multilayer perceptron neural networks. Am J Eng Applied Sci 4: 576-585, 2011.

14 Sherbet GV: Calcium signalling in cancer: The role of calcium binding proteins in cell proliferation, invasion and metastasis. CRC Press, Boca Raton, FL, USA, 2001.

15 Parker C, Whittaker PA, Usmani B, Lakshmi MS and Sherbet $\mathrm{GV}$ : Induction of $18 \mathrm{~A} 2 / \mathrm{mts} 1$ gene expression and its effects on metastasis and cell cycle control. DNA Cell Biol 13: 1021-1028, 1994.

16 Sherbet GV, Parker C, Usmani BA and Lakshmi MS: Epidermal growth factor receptor status correlates with cell proliferationrelated $18 \mathrm{~A} 2 / \mathrm{mts} 1$ gene expression in human carcinoma cell lines. Ann NY Acad Sci 768: 272-276, 1995.

17 Lloyd BH, Platt-Higgins A, Rudland PS and Barraclough R: Human S100A4 (p9Ka) induces the metastatic phenotype upon benign tumour cells. Oncogene 17: 465-473, 1998.

18 Royds JA, Stephenson TJ, Rees RC, Shorthouse AJ and Silocks PB: $n m 23$ protein expression in ductal in situ and invasive human breast carcinoma. J Natl Cancer Inst 85: 727-731, 1993.

19 Hennessy C, Henry JA, May FEB, Westley BR, Angus B and Lennard TWJ: Expression of the antimetastatic gene nm23 in human breast cancer: An association with good prognosis. J Natl Cancer Inst 83: 281-285, 1991. 
20 Tokunaga Y, Urano T, Furakawa K, Kondo H, Kanematsu T and Shiku H: Reduced expression of nm23-H1, but not of nm23-H2, is concordant with the frequency of lymph node metastasis of breast cancer. Int J Cancer 55: 66-71, 1993.

21 Grey SR, Dlay SS and Sherbet GV: Prediction of nodal spread of breast cancer by artificial neural network-based analyses of S100A4, nm23 and steroid receptor expression. Clin Exptl Metastasis 20: 507-514, 2003.

22 Grey SR, Dlay SS and Sherbet GV: Artificial neural networks in cancer progression and prognosis. In: Molecular and cellular pathology of cancer progression and prognosis. Sherbet GV (ed.). Research Signpost, Trivandrum, pp. 199-219, 2006.

23 Caronongan A III, Venturini B, Canuti D, Dlay S, Naguib RN and Sherbet GV: Neural analyses validate and emphasize the role of progesterone receptor in breast cancer progression and prognosis. Anticancer Res 36: 1909-1915, 2016.

24 Chang, SW, Abdul-Kareem S, Merican AF and Zain RB: Oral cancer prognosis based on clinicopathologic and genomic markers using a hybrid of feature selection and machine learning. BMC Bioinformatics 14: 170, 2013.

25 Tsao CW, Liu CY, Cha TL, Wu ST, Sun GH, Yu DS, Chen HI, Chang SY, Chen SC and Hsu CY: Artificial neural network for predicting pathological stage of clinically localised prostate cancer in a Taiwanese population. J Chinese Med Assoc 77: 513518, 2014.

26 Qiao G, Li J, Huang A, Yan Z, Lau WY and Shen F: Artificial neural networking model for the prediction of post-hepatectomy survival of patients with early hepatocellular carcinoma. J Gastroentrol Hepatol 29: 2014-2020, 2014.

27 Andronas M, Dlay SS and Sherbet GV: Oestrogen and progesterone receptor expression influences DNA ploidy and the proliferation potential of breast cancer cells. Anticancer Res 23: 3029-3039, 2003.

28 Naguib RNG, Mat-Sakim HA, Lakshmi MS, Wadehra V, Lennard TWJ, Bhatavdekar J and Sherbet GV: Logistic regression in the analysis of image cytometric data of fine needle aspirated cells of breast cancer patients. A comparison with artificial neural networks. Proc Conf IEEE Eng Med Soc 20: 982-985, 1998.

29 Naguib RNG, Mat-Sakim HA, Lakshmi MS, Wadehra V, Lennard TWJ, Bhatavdekar J and Sherbet GV: DNA ploidy and cell cycle distribution of breast cancer aspirate cells measured by image cytometry and analysed by artificial neural networks for their prognostic significance. IEEE Trans Inf Tech Med 3: 61-69, 1999.

30 Sherbet GV and Dlay SS: Does DNA ploidy and synthesis phase dynamic accentuate the predictive power of oestrogen and progesterone receptors in breast cancer progression and prognosis. Atlas Genet Cytogenet Oncol Haematol, 2018 http://atlasgeneticsoncology.org/Deep/DNAploidyERandPRinBre astCancerID20148.htm [Epub ahead of print]

31 Elias KM, Fendler W, Stawiski K, Fiascone SJ, Vitonis AF, Berkowitz RS, Frendl G, Konstatinopoulos P, Crum CP, Kedzierska M, Cramer DW and Chowdhury D: Diagnostic potential for a serum miRNA neural network for detection of ovarian cancer. Elife 6 pii: e28932, 2017. doi: 10.7554/eLife.28932. [Epub ahead of print]

32 Nustad K, Bast RC Jr, Brien TJ, Nilsson O, Seguin P, Suresh MR, Saga T, Nozawa S, Bormer OP, de Bruijn HW, Nap M, Vitali A, Gadnell M, Clark J, Shigemasa K, Karlsson B, Kreutz FT, Jette D, Sakahara H, Endo K, Paus E, Warren D, Hammarstrom S, Kenemans P and Hilgers J: Specificity and affinity of 26 monoclonal antibodies against the CA 125 antigen: First report from the ISOBM TD-1 workshop. Inter Soc Oncodevelop Biol Med Tumour Biol 17: 196-219, 1996.

33 Rustin GJ, Marples M, Nelstrop AE, Mahmudi M and Meyer T: Use of CA-125 to define progression of ovarian cancer in patients with persistently elevated levels. J Clin Oncol 19: 4054-4057, 2001.

34 Huang CJ, Jiang JK, Chang SC, Lin JK and Yang SH: Serum CA125 concentration as a predictor of peritoneal dissemination of colorectal cancer in men and women. Medicine (Baltimore) 95: e5177, 2016.

35 Zhu W and Kan X: Neural network cascade optimizes micro RNA biomarker selection for nasopharyngeal cancer prognosis. PloS One 9: e110537, 2014.

36 Pia A, Zhong X and Rayner S: miRAW: A deep learning-based approach to predict microRNA targets by analyzing whole microRNA transcripts. PloS Comput Biol 14: e1006185, 2018.

37 Wen M, Cong P, Zhang Z, Lu H and Li T: DeepMirTar: a deeplearning approach for predicting human miRNA targets. Bioinformatics, 2018. doi: 10.1093/bioinformatics/bty424. [Epub ahead of print]

38 Brown M and Harris C: Neurofuzzy adaptive modelling and control. Prentice Hall: New York, 1994.

39 Chen MY and Linkens DA: A systematic neuro-fuzzy modeling framework with application to material property prediction. IEEE Trans Cybern 31: 781-790, 2001.

40 Lin CT and Lee G: Neural fuzzy systems: A neural-fuzzy synergism to intelligent systems, Prentice-Hall Inc., NJ, USA, 1996.

41 Somayeh N, Teshnehlab M and Shoorehdeli MA: Breast cancer classification based on advanced multi- dimensional fuzzy neural network. J Med Systems 36: 2713-2720, 2012.

42 Fuller R: Neural fuzzy systems, 1995. Available from: uniobuda.hu/users/fuller.robert/ln1.pdf.

43 Senol C: Thyroid and breast cancer disease diagnosis using fuzzyneural networks. Proc Int Conf Elect Electronic Eng, 2009.

44 Lee S and Kil RM: Nonlinear system control based on Gaussian potential function network. Proc Conf IEEE, 1991. Available from ieexplore.ieee.org/document/187395.

45 Lilley M and Frean M: Neural Networks: A Replacement for Gaussian Processes? In: Intelligent Data Engineering and Automated Learning. Gallagher M, Hogan JP and Maire F (eds.). pp. 195-202, 2005.

46 Kuo RJ, Huang MH, Cheng WC, Lin CC and Wu YH: Application of a two-stage fuzzy neural network to a prostate cancer prognosis system. Artificial Intel Med 63: 119-133, 2015.

47 Kruse R: Fuzzy neural network. Scholarpedia 3: 6043, 2008.

48 De Castro LN and Von Zuben F: aiNET: An artificial immune network for data analysis, in Data mining: A heuristic approach. Abbas, H, Sarker R, Newton C. (eds.). Idea Group Publishing, 2001.

49 De Castro LN and Timmis J: An artificial immune network for multimodal function optimisation. Proc IEEE World Congress on Evolutionary Computation, pp. 669-674, 2002.

50 De Castro LN and Von Zuben FJ: Learning and optimisation using the clonal selection principle. IEEE T EVOLUT COMPUT 6: 239-251, 2002.

Received October 19, 2018

Revised October 30, 2018

Accepted November 2, 2018 